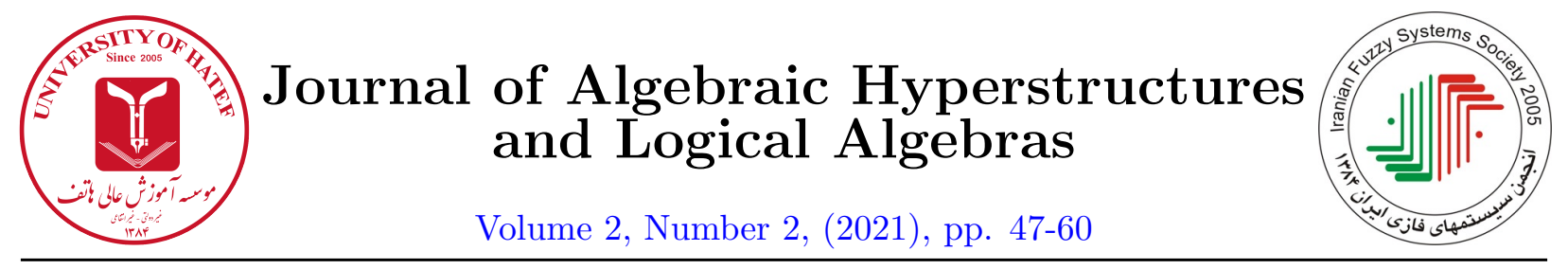

\title{
Connections between fuzzy multi-groups and fuzzy multi-graphs
}

\author{
S. Mirvakili ${ }^{1}$, H. Naraghi ${ }^{2}$ and M. Shirvani ${ }^{3}$ \\ ${ }^{1,2,3}$ Department of Mathematics, Payame Noor University, Tehran, Iran \\ saeed_mirvakili@pnu.ac.ir, ho.naraghi@pnu.ac.ir
}

\begin{abstract}
In this paper, we introduce the concept of fuzzy multigraphs and a type of isomorphism on fuzzy multi-graphs and study their properties. We show that how to assign a fuzzy multi-graph to the appropriate fuzzy multi-groups. We also prove that every fuzzy multi-group of group $G$ can be embedded into the fuzzy multi-group of the group $\operatorname{Aut}\left(\Gamma_{\wedge}^{\sigma}\right)$, where $\operatorname{Aut}\left(\Gamma_{\wedge}^{\sigma}\right)$ is a group of automorphisms of the fuzzy multi-graph $\Gamma_{\wedge}^{\sigma}$.
\end{abstract}

Article Information

Corresponding Author:

S. Mirvakili;

Received: April 2021;

Revised: May 2021;

Accepted: June 2021;

Paper type: Original.

\section{Keywords:}

Fuzzy multi-graph, fuzzy multi-group, fuzzy graph, automorphism.

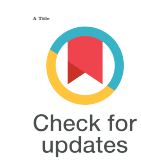

\section{Introduction}

The concept of fuzzy sets was first introduced by Zadeh in 1965 [2.9]. The study of fuzzy algebraic structures was started with the introduction of the concept of fuzzy subgroups by Rosenfeld in 1971 [21]. In 1975 Negoita and Ralescu [20], considered a generalization of Rosenfeld's definition in which the unit interval $I=[0,1]$ was replaced by an appropriate lattice structure. In 1979 Anthony and Sherwood [T] redefined a fuzzy subgroup of a group using the concept of the triangular norm. In fact, many basic properties in group theory are found to be carried over to fuzzy groups.

In the classical set theory, repetition of elements in a collection is not allowed. The notion of a group was built on this standpoint. However, the repetition of objects in real-life situations can not be ignored. This gave birth to the idea of multi-sets. The term multi-set was first suggested by N.G. De Bruijn in private correspondence to Knuth as noted in [15], as a generalization of sets (that is, in a multi-set, elements are allowed to repeat). Elaborate works on multi-set and its applications can be found in [7, 8, [27].

https://doi.org/10.52547/HATEF.JAHLA.2.2.47 
By the generalization of a set as a multi-set, then it is natural to generalize group as a multigroup. The notion of multi-group was first mentioned in [10]. Further studies on the concept of multi-groups via multi-sets can be found in [2, 16, 24].

The concept of fuzzy multi-groups was proposed in [25] as an algebraic structure of fuzzy multisets that generalized fuzzy groups. This algebraic structure is a multi-set of $G \times[0,1]$ satisfying some set of axioms, where $G$ is a classical group. In fact, since fuzzy multi-set is a generalization of fuzzy set, it follows that fuzzy multi-group is an extension of fuzzy group. The concept of fuzzy multi-groups constitutes an application of fuzzy multi-sets to the notion of group. The notion of fuzzy multi-groups and some properties of fuzzy multi-groups were explicated in [3, 12, 14]. The idea of homomorphism in fuzzy multi-groups context was extensively explored in [11, 1:3].

Rosenfield et al. [21, 22] introduced the concept of fuzzy graphs. Recently, Borzooei et al. [9] obtained some results in fuzzy graphs. Mirvakili and Naraghi [17] gave some results between hypergroups, fuzzy subgroups, and fuzzy graphs. A multi-graph [4] is a graph that may contain multiple edges between any two vertices, but it does not contain any self-loops. A generalization of multi-graphs and fuzzy graphs is fuzzy multi-graphs. To define a fuzzy planar graph, a fuzzy multi-graph is essential as planar graphs contain multi-edges. In [2:3] fuzzy multi-graph is defined. Double layered fuzzy planar graphs (DLFPG) are defined by fuzzy multi-graphs [23].

Bhutani and Bhattacharya [5, 6] gave some results of fuzzy graphs based on fuzzy subgroups. This fact motivated us to generalize the concept of fuzzy graphs to fuzzy multi-graphs. In fact, fuzzy multi-graph is a generalization of multi-graph, fuzzy graph, and graph. Moreover, there are some methods for obtaining fuzzy multi-groups from fuzzy multi-graphs. This leads us to construct some fuzzy multi-groups by fuzzy multi-graphs.

Fuzzy algebraic structures play a prominent role in mathematics with wide applications in many other branches such as theoretical physics, computer sciences, control engineering, information sciences and coding theory. Graph theory has numerous applications to problems in systems analysis, operations research, transportation, and economics. However, in many cases, some aspects of a graph-theoretic problem may be uncertain. It is natural to deal with the uncertainty using fuzzy set theory and multi-set theory. An application of fuzzy multi-groups is given by Mirvakili et al., [18]. They generated one-time passwords (OTP) by fuzzy multi-groups.

The organization of this paper is as follows: In Section 2, some preliminary definitions and concepts are given. In Section 3, we obtain a fuzzy multi-group by a fuzzy graph and a fuzzy multi-graph. In Section 4, we define the concept of isomorphism and automorphism on a fuzzy multi-graph and find several new results between fuzzy multi-graph and fuzzy multi-group by using the group of automorphism of a fuzzy multi-graph. Finally, a fuzzy multi-group is embedded in a fuzzy multi-group of the automorphism group of a fuzzy multi-graph.

\section{Preliminaries}

In this section, we study the fundamental definitions that will be used in the sequel. We use $I=[0,1]$, the real unit interval, as a chain with the usual ordering, which $\wedge$ stands for infimum (or intersection), $\vee$ stands for supremum (or union) as the degree of membership and by $I^{n}$ we mean that $I \times I \times \cdots \times I$ for $n$ times. A fuzzy subset of a set $X$ is defined as a mapping $\mu: X \rightarrow[0,1]$. Moreover, we define

$$
\alpha_{\mu}=\bigvee\{\mu(x) \mid x \in X\}, \quad \beta_{\mu}=\bigwedge\{\mu(x) \mid x \in X\}
$$

In throughout of paper, let $\mathbb{W}$ be the set of all nonnegative integers and $\mathbb{N}_{n}=\{1,2, \ldots, n\}$. 
We denote the set of all fuzzy subsets of $X$ by $[0,1]^{X}$. Further, we denote fuzzy subsets by the Greek letters $\mu, \nu, \eta$, etc. Let $\mu, \nu \in[0,1]^{X}$. If $\mu(x) \leq \nu(x)$, for all $x \in X$, then we say that $\mu$ is contained in $\nu$ (or $\nu$ contains $\mu$ ) and we write $\mu \subseteq \nu$. Let $\mu \in[0,1]^{X}$, for $\alpha \in[0,1]$, then the $\alpha$-cut (or $\alpha$-level) subset of $\mu$ denoted by $\mu_{\alpha}$ can be defined as $\mu_{\alpha}=\{x \mid x \in X, \mu(x) \geq \alpha\}$.

The fuzzy subset $\mu$ of the group $\left(G, \cdot,{ }^{-1}, e\right)$ is called a fuzzy subgroup of $G$ if:

(1) $\mu(x \cdot y) \geq \mu(x) \wedge \mu(y)$, for all $x, y \in G$;

(2) $\mu\left(x^{-1}\right) \geq \mu(x)$, for all $x \in G$.

Let $X$ be a universal set and $C_{M}: X \rightarrow \mathbb{W}$ be a function. A multi-set $M$ over the set $X$ is the following set

$$
M=\left\{\left(x, C_{M}(x)\right): x \in X, C_{M}(x)>0\right\},
$$

where the value $C_{M}(x)$ is called the multiplicity (count) of $x$ in $M$, that is, $C_{M}(x)$ is the number of copies of $x$ occur in the multi-set $M$.

Definition 2.1. [28] Let $X$ be a non-empty set. A fuzzy multi-set $A$ of $X$ is characterized by a count membership function $C M_{A}: X \rightarrow Q$, where $Q$ is the set of all multi-sets drown from the unit interval $[0,1]$.

From [26], a fuzzy multi-set can also be characterized by a high-order function. In particular, a fuzzy multi-set $A$ can be characterized by a function

$$
C M_{A}: X \rightarrow \mathbb{W}^{[0,1]} \text { or } C M_{A}: X \rightarrow[0,1] \rightarrow \mathbb{W} .
$$

By [1.9], for each $x \in X$, the membership sequence is defined as the decreasingly ordered sequence of elements in $C M_{A}(x)$ and it is denoted by:

$$
\left\{\mu_{1}(x), \mu_{2}(x), \ldots, \mu_{p}(x), \ldots: \mu_{1}(x) \geq \mu_{2}(x) \geq \cdots \geq \mu_{p}(x) \geq \ldots\right\} .
$$

Whenever the fuzzy multi-set is finite, we write

$$
C M_{A}(x)=\left(\mu_{1}(x), \mu_{2}(x), \ldots, \mu_{n}(x)\right),
$$

where $\mu_{1}(x), \mu_{2}(x), \ldots, \mu_{n}(x) \in[0,1]$ such that

$$
\mu_{1}(x) \geq \mu_{2}(x) \geq \cdots \geq \mu_{n}(x),
$$

or simply

$$
C M_{A}=\left(\mu_{i}\right)_{i \in \mathbb{N}_{n}}
$$

for $\mu_{i} \in[0,1]$. Now, a fuzzy multi-set $A$ is given as

$$
A=\left\{\frac{C M_{A}(x)}{x}: x \in X\right\} \quad \text { or } \quad A=\left\{\left(x, C M_{A}(x)\right): x \in X\right\} .
$$

In a simple term, a fuzzy multi-set $A$ of $X$ is characterized by the count membership function $C M_{A}(x)$ for $x \in X$, which takes the value of a multi-set of a unit interval $[0,1]$.

Fuzzy multi-sets are the generalization of the fuzzy sets. In fact every fuzzy set is a fuzzy multi-set but the converse is not true.

The set of all fuzzy multi-sets is depicted by $\operatorname{FMS}(X)$.

If there is no ambiguity, then we use $C M_{A}=\left(\mu_{1}, \mu_{2}, \ldots, \mu_{n}\right)$ instead of $A$. 
Example 2.2. Assume that $X=\{a, b, c\}$ is a set. Then for $C M_{A}(a)=\{1,0.5,0.4\}, C M_{A}(b)=$ $\{0.9,0.6\}$ and $C M_{A}(c)=\{0\}$, we get that $A$ is a fuzzy multi-set of $X$ written as

$$
A=\left\{\frac{(1,0.5,0.4)}{a}, \frac{(0.9,0.6)}{b}\right\} .
$$

Definition 2.3. [25] Let $\left(G, \cdot{ }^{-1}, e\right)$ be a group. A fuzzy multi-set A over $G$ is a fuzzy multi-group over $G$ if the count (count membership) of $A$ satisfies the following two conditions.

(1) $C M_{A}(x \cdot y) \geq C M_{A}(x) \wedge C M_{A}(y) \forall x, y \in G$

(2) $C M_{A}\left(x^{-1}\right) \geq C M_{A}(x) \forall x \in G$.

If there is no ambiguity, then we use $C M_{A}=\left(\mu_{1}, \mu_{2}, \ldots, \mu_{n}\right)$ over $G$ instead of multi-group A.

If there is no ambiguity, then we use xy instead of $x \cdot y$.

It can be easily verified that if $A$ is a fuzzy multi-group of $G$, then

$$
C M_{A}\left(x^{-1}\right)=C M_{A}(x) \forall x \in G .
$$

Moreover, if $e \in G$ is the identifying element of $G$, then

$$
C M_{A}(e)=\vee_{x \in G} C M_{A}(x) .
$$

The set of all fuzzy multi-groups over $G$ is depicted by $\operatorname{FMG}(G)$.

Example 2.4. Let $\left(\mathbb{Z}_{4},+\right)$ be a group. Consider

$$
A=\left\{\frac{(0.8,0.7,0.7,0.5,0.1)}{2}, \frac{(0.6,0.4,0.3,0.1)}{1}, \frac{(0.6,0.4,0.3,0.1)}{3}, \frac{(0.9,0.8,0.7,0.5,0.1)}{0}\right\}
$$

and

$$
\begin{aligned}
B=\{ & \frac{(0.6,0.4,0.3,0.1)}{2}, \frac{(0.9,0.7,0.7,0.5,0.1,0.1)}{1}, \frac{(0.8,0.7,0.7,0.5,0.1,0.1)}{3}, \\
& \left.\frac{(0.9,0.8,0.7,0.5,0.1,0.1)}{0}\right\} .
\end{aligned}
$$

Then $A$ is a fuzzy multi-group, but $B$ is not a fuzzy multi-group, because of $C M_{B}(1) \neq C M_{B}(3)$.

Definition 2.5. [5] Let $V$ be a non-empty set, $\sigma$ be a fuzzy set on $V$ and $\mu$ be a fuzzy set on $V \times V$. Then $(\sigma, \mu)$ is a fuzzy graph on $V$, if for every $x, y \in V$ we have:

(i) $\mu(x, y)=\mu(y, x)$.

(ii) $\mu(x, y) \leq \sigma(x) \wedge \sigma(y)$.

If $(V, E)$ is a graph, then set $\mu(x, y)=\left\{\begin{array}{ll}1, & x y \in E \\ 0, & x y \notin E\end{array}\right.$ and $\sigma(x)=1$ for all $x \in V$. Then $(\mu, \sigma)$ is a fuzzy graph on $V$. Also, for a fuzzy graph $(\mu, \sigma)$, we denote underlying graph $\left(V^{*}, E^{*}\right)$ where $V^{*}=\{u \mid \sigma(u)>0\}$ and $E^{*}=\{(u, v) \mid \mu(u, v)>0\}$.

Definition 2.6. [23] Let $V$ be a non-empty set and $\sigma: V \rightarrow[0,1]$ be a mapping. Moreover, let $E=\left\{\frac{\mu_{i}(x, y)}{(x, y)} \mid(x, y) \in V \times V, i \in \mathbb{N}_{n}\right\}$ be a fuzzy multi-set of $V \times V$ such that $\left(\sigma, \mu_{i}\right)$ is a fuzzy graph, for all $i \in \mathbb{N}_{n}$. Then $\Gamma=(\sigma, E)$ is denoted as a fuzzy multi-graph of $V$. 
If there is no ambiguity, then we use $\left\{\mu_{i}\right\}_{i \in \mathbb{N}_{n}}$ instead of $E$.

Example 2.7. Let $V=\{a, b, c, d, e\}$. Then Figure 1 is a fuzzy multi-graph of set $V$.

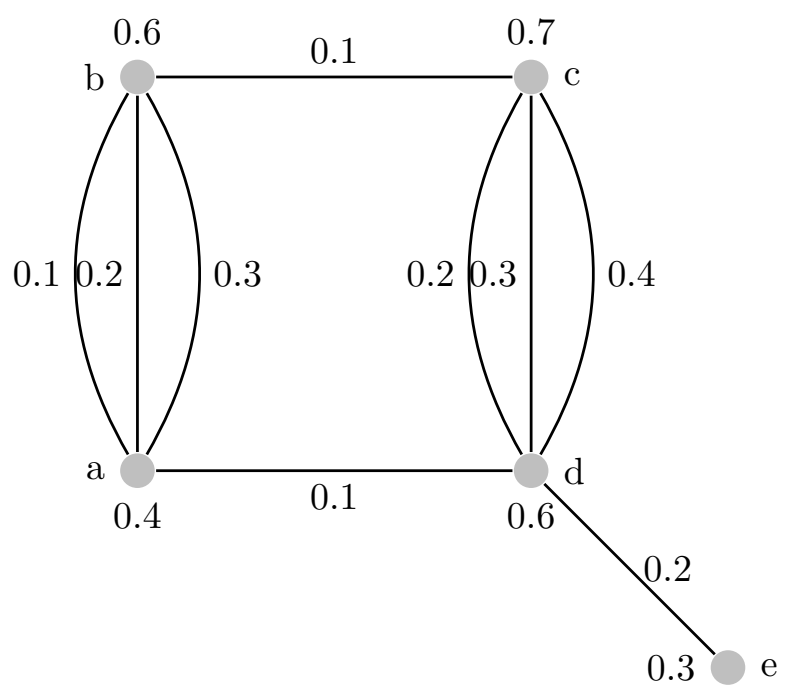

Figure 1:

\section{$3 \quad$ Fuzzy multi-groups and fuzzy multi-graphs}

In this section, we introduce the concepts of fuzzy multi-groups and fuzzy multi-graphs. By giving some results on these notions, we obtain a fuzzy multi-graph from a fuzzy multi-group.

Lemma 3.1. [18] Let $\mu$ be a fuzzy subgroup of $V$. Then $\left\{\mu_{i}\right\}_{i \in \mathbb{N}}$ is a family of fuzzy subgroups of $V$ and $\mu_{1}(x) \geq \mu_{2}(x) \geq \cdots \geq \mu_{n}(x)$ where $\mu_{n}: V \longrightarrow[0,1]$, for any $n \in \mathbb{N}$ is as follows

$$
\mu_{1}(x)=\mu(x) \quad \text { and } \quad \mu_{n}(x)=\frac{\mu_{n-1}(x)}{1+\alpha_{\mu_{n-1}}-\mu_{n-1}(x)}, \quad \text { for } \quad n \geq 2 .
$$

Corollary 3.2. Let $\mu$ be a fuzzy subgroup of $V$. If $C M_{V_{\mu}}=\left(\mu_{1}, \mu_{2}, \ldots, \mu_{n}\right)$, then $V_{\mu}=\left\{\frac{C M_{V_{\mu}}(x)}{x} \mid x \in V\right\}$ is a fuzzy multi-group over $G$.

Example 3.3. Consider the group $V=\mathbb{Z}_{4}$ and $\mu: V \longrightarrow[0,1]$ is as follows:

$$
\mu(x)= \begin{cases}1, & x \in\{0,2\} \\ \frac{1}{2}, & x \in \mathbb{Z}_{4}-\{0,2\} .\end{cases}
$$

Set $n=3$. Therefore

$$
\mu_{1}(x)=\left\{\begin{array}{ll}
1, & x \in\{0,2\} \\
\frac{1}{2}, & x \in \mathbb{Z}_{4}-\{0,2\}
\end{array}, \quad \mu_{2}(x)= \begin{cases}1, & x \in\{0,2\} \\
\frac{1}{3}, & x \in \mathbb{Z}_{4}-\{0,2\}\end{cases}\right.
$$

and

$$
\mu_{3}(x)= \begin{cases}1, & x \in\{0,2\} \\ \frac{1}{5}, & x \in \mathbb{Z}_{4}-\{0,2\}\end{cases}
$$


By the above obtained results, we have

$$
V_{\mu}=\left\{\frac{(1,1,1)}{0}, \frac{\left(\frac{1}{2}, \frac{1}{3}, \frac{1}{5}\right)}{1}, \frac{(1,1,1)}{2}, \frac{\left(\frac{1}{2}, \frac{1}{3}, \frac{1}{5}\right)}{3}\right\} .
$$

Now, we obtain some fuzzy multi-graphs from a fuzzy graph.

Proposition 3.4. Let $(\sigma, \mu)$ be a fuzzy graph on set $V$ and $n \in \mathbb{N}$. For all $x, y \in V$, define a map $\mu_{i}: V \times V \longrightarrow[0,1]$ as follows

$$
\mu_{i}(x, y)=\frac{\mu(x, y)}{1+(2+i) \alpha_{\sigma}-\mu(x, y)},
$$

and define a map $\sigma: V \longrightarrow[0,1]$ as follows

$$
\sigma^{*}(x)=\frac{\sigma(x)}{1+\alpha_{\sigma}-\sigma(x)} .
$$

Then $\left(\sigma^{*}, E=\left\{\mu_{i}\right\}_{i \in \mathbb{N}}\right)$ is a fuzzy multi-graph on $V$.

Proof. It is not difficult to see that for every $i=1,2, \ldots, n$, we have

$$
\mu_{i}(x, y)=\frac{\mu(x, y)}{1+(2+i) \alpha_{\sigma}-\mu(x, y)} \leq \frac{\sigma(x)}{1+\alpha_{\sigma}-\sigma(x)} \wedge \frac{\sigma(y)}{1+\alpha_{\sigma}-\sigma(y)}=\sigma^{*}(x) \wedge \sigma^{*}(y) .
$$

So, $\left(\sigma^{*}, E\right)$ is a fuzzy multi-graph.

Proposition 3.5. Let $C M_{V}=\left(\sigma_{1}, \sigma_{2}, \ldots, \sigma_{n}\right)$ be a fuzzy multi-group of $V$. Set $\mu_{i}(x, y)=\sigma_{i}(x) \wedge$ $\sigma_{i}(y)$ and $\sigma(x)=\sigma_{1}$. Then $\Gamma_{\wedge}^{\sigma}=\left(\sigma, E=\left\{\mu_{i}\right\}_{i \in \mathbb{N}}\right)$ is a fuzzy multi-graph of $V$, where $i \in \mathbb{N}_{n}$.

Proof. It is straightforward.

\section{Isomorphism on fuzzy multi-graphs}

In this section, we show that how to assign a fuzzy multi-graph to the appropriate fuzzy multigroups.

Definition 4.1. Let $\left(\sigma, E=\left\{\mu_{i}\right\}_{i \in \mathbb{N}_{n}}\right)$ on set $V$ and $\left(\sigma^{\prime}, E^{\prime}=\left\{\mu_{i}^{\prime}\right\}_{i \in \mathbb{N}_{n}}\right)$ on set $V^{\prime}$ be two fuzzy multi-graphs. By a homomorphism $h$ of fuzzy multi-graphs we mean a map $h: V \longrightarrow V^{\prime}$ that satisfies

1. $\sigma(x) \leq \sigma^{\prime}(h(x))$;

2. $\mu_{i}(x, y) \leq \mu_{i}^{\prime}(h(x), h(y))$,

for all $x, y \in V$. A map $h: V \longrightarrow V^{\prime}$ is called an isomorphism if $h$ is a bijective map such that $h$ and $h^{-1}$ are homomorphisms.

A homomorphism $h: V \longrightarrow V$ is called endomorphism. An isomorphism $h: V \longrightarrow V$ is called an automorphism.

Lemma 4.2. Let $\left(\sigma, E=\left\{\mu_{i}\right\}_{i \in \mathbb{N}_{n}}\right)$ on set $V$ and $\left(\sigma^{\prime}, E^{\prime}=\left\{\mu_{i}^{\prime}\right\}_{i \in \mathbb{N}_{n}}\right)$ on set $V^{\prime}$ be two fuzzy multi-graphs and $h: V \longrightarrow V^{\prime}$ be a homomorphism. Then $h$ is an isomorphism if and only if $h$ is bijective and 
1. $\sigma(x)=\sigma^{\prime}(h(x))$;

2. $\mu_{i}(x, y)=\mu_{i}^{\prime}(h(x), h(y))$,

for all $x, y \in V$.

Proof. Let $h$ be an isomorphism. Then we have

1. $\sigma(x) \leq \sigma^{\prime}(h(x))$;

2. $\mu_{i}(x, y) \leq \mu_{i}^{\prime}(h(x), h(y))$,

and

1. $\sigma^{\prime}(u) \leq \sigma\left(h^{-1}(u)\right)$;

2. $\mu_{i}^{\prime}(u, v) \leq \mu_{i}\left(h^{-1}(u), h^{-1}(v)\right)$,

for all $x, y, u, v \in V$. Let $x, y \in V$. Then there exist $u, v \in V$ such that $h(x)=u$ and $h(y)=v$. No we have

1. $\sigma^{\prime}(h(x))=\sigma^{\prime}(u) \leq \sigma\left(h^{-1}(u)\right)=\sigma\left(h^{-1}(h(x))\right)=\sigma(x)$;

2. $\mu_{i}^{\prime}(h(x), h(y))=\mu_{i}^{\prime}(u, v) \leq \mu_{i}\left(h^{-1}(u), h^{-1}(v)\right)=\mu_{i}\left(h^{-1}(h(x)), h^{-1}(h(x))\right)=\mu_{i}(x, y)$,

Conversely, $\sigma(x)=\sigma^{\prime}(h(x))$ and $\mu_{i}(x, y)=\mu_{i}^{\prime}(h(x), h(y))$ imply that $h$ and $h^{-1}$ are homomorphisms and therefore $h$ is an isomorphism.

Proposition 4.3. Let $\left(\sigma, E=\left\{\mu_{i}\right\}_{i \in \mathbb{N}_{n}}\right)$ on set $V$ and $\left(\sigma^{\prime}, E^{\prime}=\left\{\mu_{i}^{\prime}\right\}_{i \in \mathbb{N}_{n}}\right)$ on set $V^{\prime}$ be two finite fuzzy multi-graphs, $h: V \longrightarrow V^{\prime}$ be an isomorphism and $V$ and $V^{\prime}$ be two groups. Now,

(i) If $\sigma$ is a fuzzy subgroup of $V$, then $\sigma^{\prime}$ is a fuzzy subgroup of $V^{\prime}$.

(ii) If $\mu_{i}$ is a fuzzy subgroup of $V \times V$ respect to $h$, then $\mu_{i}^{\prime}$ is a fuzzy subgroup of $V^{\prime} \times V^{\prime}$.

Proof. (i) Let $x^{\prime}, y^{\prime} \in V^{\prime}$. Then there are $x, y \in V$ such that $h(x)=x^{\prime}$ and $h(y)=y^{\prime}$. Thus

$$
\begin{aligned}
\sigma^{\prime}\left(x^{\prime} y^{\prime}\right) & =\sigma^{\prime}(h(x) h(y))=\sigma^{\prime}(h(x y))=\sigma(x y) \\
& \geq \sigma(x) \wedge \sigma(y) \\
& =\sigma^{\prime}(h(x)) \wedge \sigma^{\prime}(h(y))
\end{aligned}
$$

and

$$
\begin{aligned}
\sigma^{\prime}\left(x^{\prime-1}\right) & =\sigma^{\prime}\left(h(x)^{-1}\right)=\sigma^{\prime}\left(h\left(x^{-1}\right)\right) \\
& =\sigma\left(x^{-1}\right)=\sigma(x)=\sigma^{\prime}(h(x)) \\
& =\sigma^{\prime}\left(x^{\prime}\right) .
\end{aligned}
$$

(ii) The proof is similar to the case (i).

Corollary 4.4. Let $(\sigma, E)$ on set $V$ and $\left(\sigma^{\prime}, E^{\prime}\right)$ on set $V^{\prime}$ be two finite fuzzy multi-graphs and $h: V \longrightarrow V^{\prime}$ be an isomorphism. If $V^{\prime}$ is a group and $E$ is a fuzzy multi-group on $V \times V$, then $E^{\prime}$ is a fuzzy multi-group of $V^{\prime} \times V^{\prime}$.

Proposition 4.5. Consider a fuzzy multi-graph $\Gamma=\left(\sigma, E=\left\{\mu_{i}\right\}_{i \in \mathbb{N}_{n}}\right)$ on set $V$. Let $(\operatorname{Aut}(\Gamma), \circ)$ be the set of all automorphisms of $V$. Then $(\operatorname{Aut}(\Gamma), \circ)$ is a group. 
Proof. Let $\phi, \psi \in \operatorname{Aut}(\Gamma)$ and $x, y \in V$. Then

$$
\sigma(\phi \circ \psi(x))=\sigma(\phi(\psi(x)))=\sigma(\psi(x))=\sigma(x),
$$

and

$$
\begin{aligned}
\mu_{i}(\phi \circ \psi(x), \phi \circ \psi(y)) & =\mu_{i}(\phi(\psi(x)), \phi(\psi(y))) \\
& =\mu_{i}(\psi(x), \psi(x)) \\
& =\mu_{i}(x, y) .
\end{aligned}
$$

Thus, $\phi \circ \psi \in \operatorname{Aut}(\Gamma)$. Clearly, $\operatorname{Aut}(\Gamma)$ satisfies associativity under the operation $\circ$. Also, $\phi \circ i d=\phi=i d \circ \phi$, where $i d: V \rightarrow V$ defined by $i d(x)=x$, for all $x \in V$. This shows $i d$ is an identity element. Moreover, for every $\phi \in \operatorname{Aut}(\Gamma)$,

$$
\mu_{i}\left(\phi^{-1}(x), \phi^{-1}(y)\right)=\mu_{i}\left(\phi \circ \phi^{-1}(x), \phi \circ \phi^{-1}(y)\right)=\mu_{i}(x, y)
$$

and

$$
\sigma\left(\phi^{-1}(x)\right)=\sigma\left(\phi \circ \phi^{-1}(x)\right)=\sigma(x),
$$

hence, $\phi^{-1} \in \operatorname{Aut}(\Gamma)$. Therefore $(\operatorname{Aut}(\Gamma), \circ)$ is a group.

Proposition 4.6. If two fuzzy multi-graphs $\Gamma=\left(\sigma, E=\left\{\mu_{i}\right\}_{i \in \mathbb{N}_{n}}\right)$ on set $V$ and $\Gamma^{\prime}=\left(\sigma^{\prime}, E^{\prime}=\right.$ $\left.\left\{\mu_{i}^{\prime}\right\}_{i \in \mathbb{N}_{n}}\right)$ on set $V^{\prime}$ are isomorphic, then $\operatorname{Aut}(\Gamma) \cong \operatorname{Aut}\left(\Gamma^{\prime}\right)$.

Proof. There is an isomorphism $\pi: V \longrightarrow V^{\prime}$. For every $f \in \operatorname{Aut}(\Gamma)$ we have $\pi \circ f \circ \pi^{-1} \in \operatorname{Aut}\left(V^{\prime}\right)$, because,

$$
\begin{aligned}
\sigma^{\prime}\left(\pi \circ f \circ \pi^{-1}(x)\right) & =\sigma\left(f \circ \pi^{-1}(x)\right) \\
& =\sigma\left(\pi^{-1}(x)\right) \\
& =\sigma^{\prime}(x),
\end{aligned}
$$

and

$$
\begin{aligned}
\mu_{i}^{\prime}\left(\pi \circ f \circ \pi^{-1}(x), \pi \circ f \circ \pi^{-1}(y)\right) & =\mu_{i}\left(f \circ \pi^{-1}(x), f \circ \pi^{-1}(y)\right) \\
& =\mu_{i}\left(\pi^{-1}(x), \pi^{-1}(y)\right) \\
& =\mu_{i}^{\prime}(x, y),
\end{aligned}
$$

for all $x, y \in V$. Also, it is clear that the function $\phi: \operatorname{Aut}(\Gamma) \longrightarrow \operatorname{Aut}\left(\Gamma^{\prime}\right)$ defined by $\phi(f)=$ $\pi \circ f \circ \pi^{-1}$ is a groups isomorphism. Hence, $\operatorname{Aut}(\Gamma) \cong \operatorname{Aut}\left(\Gamma^{\prime}\right)$.

The converse of Theorem 4.6 is not true:

Example 4.7. Consider two sets $V=\{a, b\}$ and $V^{\prime}=\left\{a^{\prime}, b^{\prime}\right\}$. Let $\sigma, \sigma^{\prime}$ and $\left(\sigma_{1}^{\prime}, \sigma_{2}^{\prime}\right)$ be as follows:

$$
\begin{aligned}
& \sigma(x)=\left\{\begin{array}{ll}
1, & x=a ; \\
\frac{1}{2}, & x=b
\end{array}, \quad \sigma^{\prime}(x)=\left\{\begin{array}{ll}
\frac{1}{4}, & x=a^{\prime} ; \\
\frac{1}{8}, & x=b^{\prime}
\end{array},\right.\right. \\
& \mu_{1}(x, y)=\sigma(x) \wedge \sigma(y), \quad \mu_{2}(x, y)=\frac{\sigma(x) \wedge \sigma(y)}{2}, \\
& \mu_{1}^{\prime}(x, y)=\sigma^{\prime}(x) \wedge \sigma^{\prime}(y), \quad \mu_{2}^{\prime}(x, y)=\frac{\sigma^{\prime}(x) \wedge \sigma^{\prime}(y)}{4} .
\end{aligned}
$$

Then two fuzzy multi-graphs $\Gamma=\left(\sigma, E=\left\{\mu_{i}\right\}_{i \in \mathbb{N}_{2}}\right)$ on set $V$ and $\Gamma^{\prime}=\left(\sigma^{\prime}, E^{\prime}=\left\{\mu_{i}^{\prime}\right\}_{i \in \mathbb{N}_{2}}\right)$ on set $V^{\prime}$ are not isomorphic because for every map $h: V \rightarrow V^{\prime}$ and every $x \in V, \sigma(x) \neq \sigma^{\prime}(h(x))$. But $\operatorname{Aut}(\Gamma) \cong \operatorname{Aut}\left(\Gamma^{\prime}\right) \cong\{i d\}$ 
Proposition 4.8. Let $(\sigma, E)$ on set $V$ be a fuzzy multi-graph and $c \in[0,1]$. Define a map $\tau_{c}$ : $\operatorname{Aut}(\Gamma) \longrightarrow[0,1]$ as follows

$$
\tau_{c}(f)= \begin{cases}c & f \text { is an even permutation } \\ 0 & f \text { is an odd permutation, }\end{cases}
$$

for all $f \in \operatorname{Aut}(\Gamma)$. Then $\operatorname{Aut}(\Gamma)_{\tau_{c}}$ is a fuzzy multi-group.

Proof. We prove this result in two following cases:

Case 1. Suppose $f, g \in \operatorname{Aut}(\Gamma)$ both are even or odd permutations. Thus, $f \circ g$ becomes an even permutation. Thus,

- if $f, g$ both are even permutations, then

$$
\tau_{c}(f \circ g)=c \geq \min \{c, c\}=\min \left\{\tau_{c}(f), \tau_{c}(g)\right\} .
$$

- if $f, g$ both are odd permutations, then

$$
\tau_{c}(f \circ g)=c \geq \min \{0,0\}=\min \left\{\tau_{c}(f), \tau_{c}(g)\right\} .
$$

Case 2. Suppose $f, g \in \operatorname{Aut}(\Gamma)$ such that one of them is even permutation and the other one is an odd permutation. Thus, $f \circ g$ becomes an odd permutation. Thus,

$$
0=\tau_{c}(f \circ g)=\min \{0, c\}=\min \left\{\tau_{c}(f), \tau_{c}(g)\right\} .
$$

Moreover, for all $f \in \operatorname{Aut}(\Gamma), f$ and $f^{-1}$ both are even permutations or both are odd permutations. This follows that $\tau_{c}\left(f^{-1}\right)=\tau_{c}(f)$, for all $f \in \operatorname{Aut}(\Gamma)$. Therefore, $\tau_{c}$ is a fuzzy subgroup on $\operatorname{Aut}(\Gamma)$.

Now, by Corollary 32 , we obtain $\operatorname{Aut}(\Gamma)_{\tau_{C}}$ is a fuzzy multi-group.

Proposition 4.9. Let $(\sigma, E)$ be a fuzzy multi-graph on set $V$. Set $\Theta_{m}(f)=\min \{\sigma(i): f(i) \neq i\}$ and $\Theta_{M}(f)=\max \{\sigma(i): f(i) \neq i\}$. Define a map $\psi: \operatorname{Aut}(\Gamma) \longrightarrow[0,1]$ as follows

$$
\psi(f)= \begin{cases}\frac{\Theta_{m}(f)}{1+\Theta_{M}(f)}, & f \neq i d \\ 1 & f=i d\end{cases}
$$

for all $f \in \operatorname{Aut}(\Gamma)$. Then $\operatorname{Aut}(\Gamma)_{\psi}$ is a fuzzy multi-group.

Proof. Clearly, if $f, g \in \operatorname{Aut}(\Gamma)$ and $f \circ g=\mathrm{id}$, then $\psi(f \circ g) \geq \min \{\psi(f), \psi(g)\}$. Suppose $f \circ g \neq$ id. Hence, there exists $r \in V$ such that $f \circ g(r) \neq r$ and

$$
\Theta_{m}(f \circ g)=\min \{\sigma(i): f \circ g(i) \neq i\}=\sigma(r) .
$$

We have the following two cases for $\Theta_{m}(f \circ g)$ :

Case 1. If $f \circ g(r) \neq g(r)$, then

$$
\begin{aligned}
\Theta_{m}(f \circ g) & =\sigma(r)=\sigma(g(r)) \\
& \geq \min \{\sigma(i): f(i) \neq i\}=\Theta_{m}(f) \\
& \geq \min \left\{\Theta_{m}(f), \Theta_{m}(g)\right\} .
\end{aligned}
$$


Case 2. Assume $f \circ g(r)=g(r)$. Thus, $g(r) \neq r$. Then we have

$$
\begin{aligned}
\Theta_{m}(f \circ g) & =\sigma(r) \\
& \geq \min \{\sigma(i): g(i) \neq i\}=\Theta_{m}(g) \\
& \geq \min \left\{\Theta_{m}(f), \Theta_{m}(g)\right\} .
\end{aligned}
$$

Now, Let $\Theta_{M}(f \circ g)=\sigma(t)$. We have $g(t)=t$ or $g(t) \neq t$. If $g(t)=t$, then $f(t) \neq t$ and $\sigma(t) \leq \max \{\sigma(i): f(i) \neq i\}=\Theta_{M}(f)$. If $g(t) \neq t$, then $\sigma(t) \leq\{\sigma(i): g(i) \neq i\}=\Theta_{M}(g)$. Thus we obtain

$$
\Theta_{M}(f \circ g) \leq \max \left\{\Theta_{M}(f), \Theta_{M}(g)\right\}
$$

Therefore,

$$
\psi(f \circ g) \geq \min \{\psi(f), \psi(g)\} .
$$

Moreover, $\{\sigma(i): f(i) \neq i\}=\left\{\sigma(i): f^{-1}(i) \neq i\right\}$, and so $\psi\left(f^{-1}\right)=\psi(f)$.

\section{A fuzzy multi-group embedded in a fuzzy multi-group of $\operatorname{Aut}(\Gamma)$}

In this section, we show that a finite fuzzy multi-group has an embedding into the finite fuzzy multi-group of the group of automorphisms of some fuzzy multi-graphs.

Definition 5.1. Let $V$ and $V^{\prime}$ be two finite groups. We say a fuzzy multi-group $C M_{A}=\left(\sigma_{1}, \ldots, \sigma_{n}\right)$ has an embedding into a fuzzy multi-group $C M_{A^{\prime}}=\left(\sigma_{1}^{\prime}, \ldots, \sigma_{n}^{\prime}\right)$ if there exists a one-to-one map $h: V \rightarrow V^{\prime}$ such that $C M_{A}(x) \leq C M_{A^{\prime}}(h(x))$.

Example 5.2. Consider two groups $V=\mathbb{Z}_{2}$ and $V^{\prime}=\mathbb{Z}_{4}$. Let $C M_{A}=\left(\sigma_{1}, \sigma_{2}\right)$ and $C M_{A^{\prime}}=$ $\left(\sigma_{1}^{\prime}, \sigma_{2}^{\prime}\right)$ be as follows:

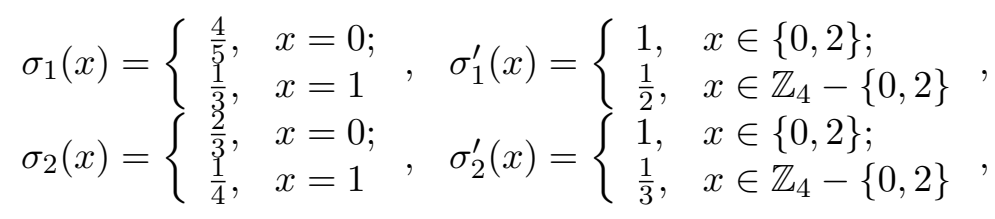

Set $h: V \rightarrow V^{\prime}$ by $h(x)=2 x$. Then $h$ is an embedding of $C M_{A}$ to $C M_{A^{\prime}}$.

Proposition 5.3. Let $\Gamma=(\sigma, E)$ be a fuzzy multi-graph of finite group $V$. Define the mappings $\tau_{i}: \operatorname{Aut}(\Gamma) \longrightarrow[0,1]$

$$
\tau_{i}(f)= \begin{cases}\alpha_{\sigma_{i}} & f \text { is an odd permutation } \\ \frac{\alpha_{\sigma_{i}}+1}{2} & f \text { is an even permutation, }\end{cases}
$$

where $i=1,2, \ldots, n, f \in \operatorname{Aut}(\Gamma)$ and $\sigma_{i}(x)=\sup _{y \in V} \mu_{i}(x, y)$. Then $C M_{\mathbf{A}(\boldsymbol{\Gamma})}=\left(\left\{\tau_{i}\right\}_{i \in \mathbb{N}}\right)$ is a fuzzy multi-group.

Proof. We prove this result in two following cases:

Case 1. Suppose $f, g \in \operatorname{Aut}(\Gamma)$ both are even or odd permutations. Thus, $f \circ g$ becomes an even permutation. Thus, if $f, g$ both are odd permutations, then

$$
\begin{aligned}
\tau_{i}(f \circ g) & =\frac{\alpha_{\sigma_{i}}+1}{2} \\
& \geq \min \left\{\alpha_{\sigma_{i}}, \alpha_{\sigma_{i}}\right\} \\
& =\min \left\{\tau_{i}(f), \tau_{i}(g)\right\} .
\end{aligned}
$$


Moreover, if $f, g$ both are even permutations, then

$$
\begin{aligned}
\tau_{i}(f \circ g) & =\frac{\alpha_{\sigma_{i}}+1}{2} \\
& \geq \min \left\{\frac{1+\alpha_{\sigma_{i}}}{2}, \frac{1+\alpha_{\sigma_{i}}}{2}\right\} \\
& =\min \left\{\tau_{i}(f), \tau_{i}(g)\right\} .
\end{aligned}
$$

Case 2. Suppose $f, g \in \operatorname{Aut}(\Gamma)$ such that one of them is an even permutation and the other one is an odd permutation. Thus, $f \circ g$ becomes an odd permutation. Then,

$$
\alpha_{\sigma_{i}}=\tau_{i}(f \circ g) \geq \min \left\{\frac{\alpha_{\sigma_{i}}+1}{2}, \alpha_{\sigma_{i}}\right\} .
$$

Also, for all $f \in \operatorname{Aut}(\Gamma), f$ and $f^{-1}$ both are even permutations or both are odd permutations. This follows that $\tau_{i}\left(f^{-1}\right)=\tau_{i}(f)$, for all $f \in \operatorname{Aut}(\Gamma)$. Therefore, $\tau_{i}$ is a fuzzy subgroup on Aut $(\Gamma)$, so $C M_{\mathbf{A}(\boldsymbol{\Gamma})}=\left(\left\{\tau_{i}\right\}_{i \in \mathbb{N}}\right)$ is a fuzzy multi-group on the group $\operatorname{Aut}(\Gamma)$.

Proposition 5.4. A bijective endomorphism of finite multi-graph $(\sigma, E)$ on $V$ is an automorphism.

Proof. It is straightforward.

Proposition 5.5. Every fuzzy multi-group has an embedding into the finite fuzzy multi-group of the group of automorphisms of some fuzzy multi-graphs.

Proof. Let $C M_{A}=\left(\left\{\sigma_{i}\right\}_{i \in \mathbb{N}_{n}}\right)$ be a fuzzy multi-group. Then by Proposition 4.3 , $\Gamma_{\wedge}^{\sigma}$ is a fuzzy multi-graph.

Let $a \in V$ such that $\alpha_{\sigma}=\sigma(a)$ and define $h: V \rightarrow \operatorname{Aut}\left(\Gamma_{\wedge}^{\sigma}\right)$ by $h(u)=h_{u}$ when $h_{u}(x)=a x$, for all $x \in V$. Then $h_{u}$ is a bijective endomorphism because

$$
\sigma\left(h_{u}(x)\right)=\sigma(a x) \geq \sigma(x) \wedge \sigma(a)=\sigma(x) .
$$

Moreover,

$$
\begin{aligned}
\mu_{i}\left(h_{u}(x), h_{u}(y)\right) & =\sigma_{i}\left(h_{u}(x)\right) \wedge \sigma_{i}\left(h_{u}(y)\right) \\
& =\sigma_{i}(a x) \wedge \sigma_{i}(a y) \\
& \geq \sigma_{i}(x) \wedge \sigma_{i}(y)
\end{aligned}
$$

Therefore, according to Proposition 5.4, $h_{u}$ is an automorphism. Given this inequality $\frac{1+\alpha_{\sigma_{i}}}{2} \geq$ $\alpha_{\sigma_{i}}$. Hence

$$
\tau_{i}\left(h_{u}\right) \geq \alpha_{\sigma_{i}} \geq \sigma_{i}(u)
$$

for all $u \in V$. Thus $C M_{\mathbf{A}\left(\boldsymbol{\Gamma}_{\wedge}^{\sigma}\right)}=\left(\left\{\tau_{i}\right\}_{i \in \mathbb{N}_{n}}\right)$ is a fuzzy multi-group on the group $A u t\left(\Gamma_{\wedge}^{\sigma}\right)$. Therefore $C M_{A}=\left(\left\{\sigma_{i}\right\}_{i \in \mathbb{N}_{n}}\right)$ has an embedding in $C M_{\mathbf{A}\left(\boldsymbol{\Gamma}_{\wedge}^{\sigma}\right)}=\left(\left\{\tau_{i}\right\}_{i \in \mathbb{N}_{n}}\right)$.

Example 5.6. Consider the group $V=\left(\mathbb{Z}_{3},+\right)$. Let $C M_{A}$ be a multi-group on the group $V$ as follows:

$$
C M_{A}=\left\{\frac{(0.6,0.4,0.3)}{2}, \frac{(0.6,0.4,0.3)}{1}, \frac{(0.9,0.8,0.7)}{0}\right\} .
$$


Then By Proposition 4.3 , for all $x, y \in V$, we have $\mu_{1}(x, y), \mu_{2}(x, y), \mu_{3}(x, y)$ as follows.

\begin{tabular}{|c|c|c|c|c|c|c|c|c|c|c|c|}
\hline$\mu_{1}$ & 0 & 1 & 2 & $\mu_{2}$ & 0 & 1 & 2 & $\mu_{3}$ & 0 & 1 & 2 \\
\hline 0 & 0.9 & 0.6 & 0.6 & 0 & 0.8 & 0.4 & 0.4 & 0 & 0.7 & 0.3 & 0.3 \\
\hline 1 & 0.6 & 0.6 & 0.6 & & 0.4 & 0.4 & 0.4 & 1 & 0.3 & 0.3 & 0.3 \\
\hline ? & 0.6 & 0.6 & 0.6 & 2 & 0.4 & 0.4 & 0.4 & 2 & 0.3 & 0.3 & 03 \\
\hline
\end{tabular}

Set $\sigma(x)=\sigma_{1}(x)$ and so $\sigma(x)=\left\{\begin{array}{ll}0.9 & x=0 \\ 0.6 & x=1,2\end{array}\right.$. It easy to see that $P(V, \mu)=(\sigma, E)$ is a fuzzy multi-graph. By the proof of Proposition 5.5, we have $a=0$, and $h_{u}(x)=0+x=x$ for all $u \in V$. Also the group of automorphisms of the fuzzy multi-graph $\Gamma_{\wedge}^{\sigma}$ is $\operatorname{Aut}\left(\Gamma_{\wedge}^{\sigma}\right)=\{i d,(12)\}$, when (12) is a permutation $\left(\begin{array}{lll}0 & 1 & 2 \\ 0 & 2 & 1\end{array}\right)$, and $\tau_{1}(f)=\left\{\begin{array}{ll}0.95 & f=i d \\ 0.9 & f=(12)\end{array}, \tau_{2}(f)=\left\{\begin{array}{ll}0.85 & f=i d \\ 0.8 & f=(12)\end{array}\right.\right.$, $\tau_{3}(f)=\left\{\begin{array}{ll}0.75 & f=i d \\ 0.7 & f=(12)\end{array}\right.$. Then $\tau_{i}\left(h_{u}\right) \geq \sigma_{i}(u)$ and this shows that the fuzzy multi-group $C M_{A}$ is embedded in the fuzzy multi-group $C M_{\mathbf{A}\left(\boldsymbol{\Gamma}_{\wedge}^{\sigma}\right)}=\left(\left\{\tau_{i}\right\}_{i \in \mathbb{N}_{3}}\right)$.

\section{Conclusion}

In this work, we obtained some results in fuzzy multi-groups and fuzzy multi-graphs. We presented a fuzzy multi-group of a fuzzy multi-graph. We introduced the notion of automorphisms on multigraphs and described a fuzzy multi-group with the underlying group of automorphisms from a fuzzy multi-graph. Finally, we proved that every fuzzy multi-group can be embedded in a fuzzy multi-group of the group of automorphisms of some fuzzy multi-graphs.

For future work, it will be interesting to introduce a $t$-norm over a fuzzy multi-group and a fuzzy multi-graph and obtain a $t$-norm fuzzy multi-group and a $t$-norm fuzzy multi-graph.

Moreover, it will be interesting to introduce the idea of this paper on multi-hypergroups and multi-hypergraphs.

\section{References}

[1] J.M. Antony, H. Sherwood, Fuzzy groups redefined, Journal of Analysis and Applications, 69 (1979), 124-130.

[2] J.A. Awolola, A.M. Ibrahim, Some results on multi-groups, Quasigroups and Related Systems, $24(2)$ (2016), 169-177.

[3] A. Baby, T.K. Shinoj, J.J. Sunil. On abelian fuzzy multi-groups and order of fuzzy multigroups, Journal of New Theory, 5(2) (2015), 80-93.

[4] V.K. Balakrishnan, Graph theory, McGraw-Hill, 1997.

[5] P. Bhattacharya, Some remarks on fuzzy graphs, Pattern Recognition Letters, 6 (1987), 297302.

[6] K.R. Bhutani, On automorphisms of fuzzy graphs, Pattern Recognition Letters, 9 (1989), 159-162.

[7] W.D. Blizard, multi-set theory, Notre Dame Journal of Formal Logic, 30 (1989), 36-66. 
[8] W.D. Blizard, The development of multi-set theory, Modern Logic, 1 (1991), 319-352.

[9] R.A. Borzooei, B. Sheikh Hoseini, M. Mohseni Takallo, Results on t-fuzzy graphs, New Mathematics and Natural Computation, 16 (2020), 143-161.

[10] M. Dresher, O. Ore, Theory of multi-groups, American Journal of Mathematics, 60 (1938), 705-733.

[11] P.A. Ejegwa, Homomorphism of fuzzy multi-groups and some of its properties, Applications and Applied Mathematics, 13(1) (2018), 114-129.

[12] P.A. Ejegwa, On fuzzy multi-groups and fuzzy submulti-groups, Journal of Fuzzy Mathematics, 26(3) (2018), 641-654.

[13] P.A. Ejegwa, On alpha-cuts homomorphism of fuzzy multi-groups, Annals of Fuzzy Mathematics and Informatics, 19(1) (2020), 73-87.

[14] P.A. Ejegwa, Some group's theoretic notions in fuzzy multi-group context, In: Jana, C., Senapati, T. Pal, M. (Eds.); Handbook of Research on Emerging Applications of Fuzzy Algebraic Structures, pp. 34-62, IGI Global Publisher, Hershey, Pennsylvania 17033-1240, USA, (2020).

[15] D. Knuth, The art of computer programming, semi numerical algorithms, Second Edition 2, Addison-Wesley, Reading, Massachusetts, 1981.

[16] L. Mao, Topological multi-groups and multifields, International Journal of Mathematics and Combinatorics, 1 (2009), 8-17.

[17] S. Mirvakili, H. Naraghi, Connected between reversible regular hypergroups, $t$-fuzzy subgroups and t-fuzzy graphs, Algebraic Hyperstructures and Logical Algebras, 1(4) (2020), 71-82.

[18] S. Mirvakili, H. Naraghi, M. Shirvani, P. Ghiasvand, Some fuzzy multi-groups obtained from fuzzy subgroups, Journal of Mathematical Extension, to appear.

[19] S. Miyamoto, K. Mizutani, multi-set model and methods for nonlinear document clustering for information retrieval, Springer-Verlag Berlin Heidelberg, (2004), 273-283.

[20] C.V. Negoita, D.A. Ralescu, Applications of fuzzy sets to system analysis, Wiley, New York, 1975 .

[21] A. Rosenfeld, Fuzzy groups, Journal of Mathematics and Applications, 35 (1971), 512-517.

[22] A. Rosenfield, Fuzzy sets and their applications, Academic Press, New York, 1975.

[23] S. Samanta, A. Pal, M. Pal, New concepts of fuzzy planar graphs, International Journal of Advanced Research in Artificial Intelligence, 3(1) (2014), 52-59.

[24] B.M. Schein, multi-groups, Journal of Algebra, 111 (1987), 114-132.

[25] T.K. Shinoj, A. Baby, J.J. Sunil, On some algebraic structures of fuzzy multi-sets, Annals of Fuzzy Mathe-matics and Informatics, 9(1) (2015), 77-90.

[26] A. Syropoulos, On generalized fuzzy multi-sets and their use in computation, Iranian Journal of Fuzzy Systems, 9(2) (2012), 113-125 
[27] N.J. Wildberger, A new look at multi-sets, School of Mathematics, UNSW Sydney 2052, Australia, 2003.

[28] R.R. Yager, On the theory of bags, International Journal of General Systems, 13 (1986), 23-37.

[29] L.A. Zadeh, Fuzzy sets, Information and Control, 8(3) (1965), 338-353. 